\title{
Acute Lassa Virus Encephalitis with Lassa Virus in the Cerebrospinal Fluid but Absent in the Blood: A Case Report with a Positive Outcome
}

\author{
Peter O. Okokhere ${ }^{a-c} \quad$ Cyril O. Erameh ${ }^{a} \quad$ Francis Alikah $^{a}$ \\ Peter E. Akhideno ${ }^{a} \quad$ Christopher O. Iruolagbe ${ }^{a} \quad$ Omoregie O. Osazuwa ${ }^{a}$ \\ Idowu A. Bankole ${ }^{a}$ Donatus I. Adomeh ${ }^{b}$ Ikponmwosa Odiab \\ Chris O. Aire $^{b}$ Jennifer Oyakhilome ${ }^{b}$ Jacqueline Agbukor ${ }^{b}$ \\ Rebecca O. Atafo ${ }^{d}$ Danny A. Asogun ${ }^{b}$ Christian T. Happi ${ }^{\mathrm{e}}$ \\ Pardis C. Sabeti ${ }^{f}$ Stephan Güntherg, h George O. Akpede ${ }^{c, i}$ \\ aDepartment of Medicine, Irrua Specialist Teaching Hospital, Irrua, Nigeria; 'b Institute of \\ Lassa Fever Research and Control, Irrua Specialist Teaching Hospital, Irrua, Nigeria; \\ 'Faculty of Clinical Sciences, Ambrose Alli University, Ekpoma, Nigeria; 'Lassa Fever Ward, \\ Irrua Specialist Teaching Hospital, Irrua, Nigeria; ePostgraduate School, Redeemer's \\ University, Ede, Nigeria; 'Department of Organismic and Evolutionary Biology, Broad \\ Institute of MIT and Harvard University, Cambridge, MA, USA; ${ }^{\text {BBernhard-Nocht Institute }}$ \\ of Tropical Medicine, Hamburg, Germany; ${ }^{\mathrm{h}}$ German Centre for Infection Research (DZIF), \\ Partner site Harmburg, Hamburg, Germany; 'Department of Paediatrics, Irrua Specialist \\ Teaching Hospital, Irrua, Nigeria
}

\section{Keywords}

Lassa virus · Encephalitis · Ribavirin · Nigeria 


\section{Case Reports in Neurology}

\begin{abstract}
It is rare both to have the central nervous system (CNS) as the main focus in the acute phase of Lassa fever infection without associated bleeding, and to find Lassa virus (LAV) in the cerebrospinal fluid (CSF) but not in the serum. We report the case of a 38-year-old Nigerian woman with mainly CNS manifestation of Lassa fever. She was admitted twice within 11 days because of persistent fever. A clinical diagnosis of acute LAV encephalitis was made because of a high index of suspicion and CNS involvement confirmed by positive reverse transcriptase polymerase chain reaction (RT-PCR) for LAV in the CSF, while her blood was repeatedly negative for LAV by RT-PCR test. She recovered fully following supportive care coupled with treatment with an 18-day course of ribavirin, and suffered no long-term neurological complication or relapse. Post-treatment CSF examination by RT-PCR did not detect LAV.
\end{abstract}

(C) 2018 The Author(s)

Published by S. Karger AG, Basel

\title{
Introduction
}

Lassa fever (LF) is endemic in Nigeria, particularly in Edo State [1]. In severe cases, LF may present with central nervous system (CNS) features, when it often indicates the late stage of the disease [2-5], and is usually associated with bleeding and poor prognosis [5].

CNS involvement without associated bleeding has been reported in Dengue fever [6], but there is limited data on the CNS being the main focus of clinical infection in LF. It is rare to find Lassa virus (LAV) in the cerebrospinal fluid (CSF) when it is absent in the serum in patients with CNS involvement [7]. To the best of our knowledge, only one case has been published of LF involving the CNS where LAV was found in the CSF but not in the serum [7]. The diagnosis was made retrospectively shortly after the patient had, unfortunately, succumbed to the disease. The patient was not treated with ribavirin because LF was not suspected clinically.

We report the case of acute LF in a 38-year-old Nigerian woman who had mainly CNS involvement without any bleeding episode. LF was suspected clinically and confirmed by positive reverse transcriptase polymerase chain reaction (RT-PCR) test for LAV in the CSF, even though the serum was repeatedly negative for LAV by RT-PCR testing. She recovered fully without any long-term neurological sequelae, following treatment with ribavirin.

\section{Case Report}

A 38-year-old Nigerian woman, resident in Ekpoma, Edo State, Nigeria, presented to our outpatient department on December 27, 2013, with a 4-day history of high-grade fever that was associated with chills and headaches. She had previously taken some doses of an antimalarial drug, but when she did not get better, she came to the hospital. There was no history of sore throat, vomiting, diarrhoea, mucosal bleeding, neck pain, or neck stiffness. Her axillary temperature and blood pressure were $39.5^{\circ} \mathrm{C}$ and $120 / 80 \mathrm{~mm} \mathrm{Hg}$, respectively. The pulse rate was $96 \mathrm{bpm}$ and the respiratory rate $24 \mathrm{cycles} / \mathrm{min}$. Although she denied any recent contact with a confirmed case of LF, she was a caregiver, about 6 months earlier, to her twin sister 
Okokhere et al.: Acute Lassa Virus Encephalitis with Lassa Virus in the CSF but Absent in the Blood

who had a prolonged febrile illness ( 3 weeks) that was complicated by severe sensorineural deafness in the acute phase.

LF was suspected because there was an outbreak in Ekpoma at the time, and she was admitted for evaluation. Her blood smear showed scanty Plasmodium falciparum while the blood sample taken on the day of arrival was negative for LAV when tested using RT-PCR. Also, the blood culture performed 2 days later yielded no bacterial growth even after 7 days of incubation.

Physical examination was unremarkable, except for two right submandibular lymph nodes, measuring $1 \mathrm{~cm} \times 1.5 \mathrm{~cm}$ each. She was treated for malaria with artemisinin-based combination therapy because her blood film smear showed $P$. falciparum parasitaemia, and because malaria is a common cause of fever in our environment. She was also given parenteral cephalosporin (Ceftriaxone) and metronidazole for suspected septicaemia. Although her temperature remained elevated $\left(38.6^{\circ} \mathrm{C}\right)$, about 8 days after admission, she was discharged on her request on January 3,2014 , when she reported being improved, and a repeat blood smear for malaria parasites taken on December 31, 2013 was negative. She was given oral quinine tablets, doxycycline, and cefuroxime to take at home.

The patient was rushed back to the hospital 2 days after discharge (January 5, 2014) when she was observed to be behaving irrationally at home. She had remained febrile, with highgrade fever, while at home. At the time of arrival, her axillary temperature was $38.4^{\circ} \mathrm{C}$, pulse rate was $96 \mathrm{bpm}$, blood pressure was $120 / 70 \mathrm{~mm} \mathrm{Hg}$, and respiratory rate was $24 \mathrm{cycles} / \mathrm{min}$. There were no signs suggestive of meningeal irritation, but she was irritable, confused, and aphasic. A clinical diagnosis of acute viral encephalitis was made, and she was readmitted.

For the second time since the onset of illness, another blood sample tested negative for LAV using the RT-PCR method on the 1st day of readmission. On the following day, blood samples sent for serological tests for IgM and IgG to LAV came out positive (Table 1). A CSF sample was also collected by lumber puncture the following day and sent for biochemical and microbiological analysis. An aliquot of the CSF sample was tested for the presence of LAV by the RTPCR method: the result was positive (Table 1). Other findings on CSF examination were pleocytosis (CSF white blood cell count of 300 cells $/ \mathrm{mm}^{3}$ ) with differential of $20 \%$ neutrophils and $80 \%$ lymphocytes, but normal CSF protein $(35 \mathrm{mg} / \mathrm{dL})$ and glucose $(55 \mathrm{mg} / \mathrm{dL}$ or $59.1 \%$ of the simultaneous blood glucose level of $93 \mathrm{mg} / \mathrm{dL}$ ) levels. Gram stain of the CSF was negative and the culture sterile.

Serological tests for HIV I and II, hepatitis B surface antigen, and hepatitis C core were negative, as were blood and urine cultures for bacteria. Urinalysis showed proteinuria $(3+)$ and microscopic haematuria (2+). Her serum aminotransferases and alkaline phosphatise were elevated; she had slightly elevated total and conjugated bilirubin levels (Table 2). An abdominal ultrasound scan was normal, while a PCR-based pan-flavivirus assay excluded Dengue and Yellow fever. The haematocrit and haemoglobin levels, and serum electrolytes, urea, and creatinine were normal during both admissions. There was leucocytosis, but no neutrophilia on readmission. CT or MRI brain scan was not done because the facilities were not available in our centre at the time.

After completing the initial clinical assessment in the emergency room, an intravenous line was inserted in a peripheral vein and intravenous fluids given. Supplemental oxygen was administered through nasal prongs. Sedation with parenteral diazepam $(5 \mathrm{mg})$ was given to 
control her restlessness and irritability, and allow a urethral catheter and nasogastric tube (NGT) to be inserted. Parenteral dexamethasone ( 8 mg every $8 \mathrm{~h}$ ) was started.

Ribavirin, the specific antiviral drug for treating LF, was commenced on the 2nd day (January 6,2014 ) of readmission, a day before the detection of LAV in the CSF, and it was continued for 18 days.

For the next $48 \mathrm{~h}$ after readmission, the patient's clinical condition worsened, with deepening level of unconsciousness (GCS 7). She was more restless, and her peak temperature on the 2 nd day of readmission was $39.0^{\circ} \mathrm{C}$. Feeding (fluid diet) and oral drugs were administered via NGT. By the 3rd day, the patient's restlessness had significantly subsided; she was conscious but unable to engage in conversation, responding to command only by nodding her head, and the maximum recorded (peak) temperature was $38.1^{\circ} \mathrm{C}$.

She was observed to be responding appropriately to verbal commands and questions by the 5th day of readmission. A day later, the NGT was removed and oral feeding was started, because her sensorium had significantly improved, and she was able to tolerate meals. She was now able to speak and converse normally for the first time since readmission. Her maximum axillary temperature dropped gradually, and was $37.1^{\circ} \mathrm{C}$ by day 7 , the day she was observed to have hand tremors.

The clinical status of the patient continued to improve; she became stronger, was eating well, became ambulant, and her temperature measurements were within normal limits (Fig. 1). She had tinnitus with associated mild bilateral hearing impairment which started on the 16 th day of admission and lasted for 3 days. She was discharged on the morning of January 23,2014 , in satisfactory clinical condition.

A CSF sample taken 5 days after discharge was negative for LAV by RT-PCR. Regular periodic clinical neurological reviews for 32 months after discharge showed no long-term neurological complication or clinical relapse, and she has remained well, carrying out her domestic and official duties in good health.

\section{Discussion}

Outbreaks of LF have been documented in Ekpoma, Nigeria, and its environs [1], where the reservoir rodent, Mastomys natalensis, is endemic [8].

Our patient, who was resident in Ekpoma, Nigeria, developed encephalitis about 11 days into her febrile illness with CNS features that included confusion, disorientation, restlessness, irritability, aphasia, transient bilateral 8th nerve deafness, and altered level of consciousness, but without meningeal involvement. There was blood evidence of liver function derangement, but she was not clinically jaundiced; the serum electrolytes, urea, creatinine, blood sugar, and haemoglobin levels were normal, thus making encephalopathy from a metabolic cause an unlikely diagnosis.

Although acute LF was suspected on initial presentation, the diagnosis was not confirmed. Fortunately, even though it was still not confirmed by RT-PCR testing of the blood on readmission, treatment with ribavirin was commenced because of a strong index of suspicion, a decision that probably played a significant role in the positive outcome. 
Okokhere et al.: Acute Lassa Virus Encephalitis with Lassa Virus in the CSF but Absent in the Blood

Delay in the diagnosis of LF because of a low index of suspicion is not an uncommon phenomenon in West Africa, especially when malaria parasites are demonstrated in febrile patients [9], sometimes resulting in fatalities [9]. LF has been shown to coexist with malaria in a significant number of individuals in malaria/LF endemic areas [10]. Furthermore, the findings of proteinuria and haematuria in our patient are not unusual features in LF patients in our environment [11], and their presence in the urine should heighten the suspicion of LF in febrile patients in LF endemic areas [11].

Though it is known that bleeding is seen in less than $20 \%$ of cases of acute LF [2], many health care workers rarely consider acute LF when the patient does not present with bleeding $[7,9]$. This can lead to a delay in diagnosis and consequent fatality $[7,9]$. Our case shows that even severe cases of LF may not have bleeding as a clinical feature.

As a diagnostic tool, RT-PCR testing for LAV compares favourably with positive viral culture of blood [12]. Because culturing of the LAV is done in a BL4 laboratory, which is not available in Nigeria, and it takes about a week to complete, RT-PCR is considered adequate for confirming a diagnosis of LF in the early phase of the disease [12] when treatment is most effective [2]. As shown by Günther et al. [7], as well as this case, LAV may not be detected in the blood in all cases. In such situations, a high index of suspicion is required to make a clinical diagnosis of LF, which must be confirmed by demonstrating the presence of LAV in the appropriate body fluid.

It is not clear why LAV is detected in body fluids and not in serum in some cases of LF. It is probable that the LAV that circulates beyond the blood space into other body fluids in certain individuals survives or escapes the activity of the immune system, and LAV might be relatively shielded from destruction in body fluids that are not sera. The presence of LAV-specific IgM and IgG found in the serum of the case reported by Gunther et al. [7], and in this case, may indicate that the virus was present in the blood at some point during the course of the illness, but it was cleared by the blood-based immune system - either completely or to a level too low to be detected by RT-PCR.

The mechanism of CNS disease in LF may be immunological, cytopathic, or both [7, 12]. A growing body of evidence suggests that direct invasion of the CNS tissues by LAV may be important in the pathogenesis of the disease $[7,13]$. Our case adds to that body of evidence, especially with the demonstration of evidence of LAV in the CSF. More research is needed to clearly elucidate the neuropathogenic mechanism in CNS LF.

The CNS is involved in severe cases of $\operatorname{LF}[2,4,10]$. CNS manifestations in the acute phase include seizures, coma, confusion, disorientation, restlessness, irritability, and sensorineural deafness [2, 4]; late features are ataxia [2] and psychiatric syndromes [5].

LF affecting the CNS is usually associated with high mortality rates $[4,10,14]$. Our case report would suggest that ribavirin therapy may be life-saving in CNS LAV infection, when treatment is commenced as soon as it is suspected clinically, even before a definitive laboratory diagnosis is made.

This report supports the recommendation of Günther et al. [7] that LF be considered in the differential diagnoses of encephalopathy/encephalitis in febrile patients in LF endemic areas, and in patients with fevers imported from LF endemic regions with associated CNS manifestation, even when bleeding is not a feature. Our findings further emphasize the relevance of having a high index of suspicion for LF in patients with persistent fever even when initial RT-PCR blood testing is negative for LAV in endemic areas. Our report should further 
alert physicians practising in West Africa to the fact that malaria and LF can coexist in the same patient, and that the presence of a positive blood smear for malaria does not exclude LF as a cause of CNS disease in areas where both are endemic.

Furthermore, the long-term follow-up result of this case also might indicate that, unlike Ebola encephalitis [15], relapse after successful treatment of LAV encephalitis may be an unlikely clinical phenomenon. However, long-term follow-up of more cases of treated LAV encephalitis is required to confirm this.

\section{Conclusion}

Our case report shows that LF patients can present acutely with mainly severe CNS features in areas endemic for the disease. In such a situation, a high index of suspicion is required to make the clinical diagnosis. Laboratory detection by RT-PCR of LAV in the CSF, especially when LAV is undetected in the serum, is confirmatory of CNS disease. Treatment with ribavirin may be life-saving, and curative when started as soon as CNS features are first observed.

\section{Limitations}

The main limitations of the study include our inability to carry out brain imaging of the patient and detailed virology of the causative virus.

\section{Acknowledgements}

We thank our patient who graciously consented to having her case reported.

This work received support from the German Research Foundation (DFG; grant GU883/41), the World Bank project (grant ACE 019), the NIH through H3Africa project (grant 5U01HG007480-03), and the German Centre for Infection Research. The study design, data collection, analysis and interpretation, and manuscript writing were not interfered with by the funding agencies.

\section{Statement of Ethics}

The patient's consent and the approval of the Irrua Specialist Teaching Hospital Research and Ethics Committee were obtained.

\section{Disclosure Statement}

The authors declare no conflict of interest. 
Okokhere et al.: Acute Lassa Virus Encephalitis with Lassa Virus in the CSF but Absent in the Blood

\section{Author Contributions}

All authors had unhindered access to the data, and final responsibility to submit the manuscript for publication is that of the corresponding author.

\section{References}

1 Asogun DA, Adomeh DI, Ehimuan J, Odia I, Hass M, Gabriel M, et al. Molecular diagnostics for Lassa fever at Irrua specialist teaching hospital, Nigeria: lessons learnt from two years of laboratory operation. PLoS Negl Trop Dis. 2012;6(9):e1839.

2 McCormick JB, King IJ, Webb PA, Johnson KM, O’Sullivan R, Smith ES, et al. A case-control study of the clinical diagnosis and course of Lassa fever. J Infect Dis. 1987 Mar;155(3):445-55.

3 Enria D, Mills JN, Flick R, et al. Arena Viruses. In: Guerrant RL, Walker DH, Weller PF, editors. Tropical Infectious Diseases: Principles, Pathogens and Practice. 2nd ed. Philadelphia (PA): Elsevier Churchill Livingstone; 2006. pp. 734-55.

4 Okokhere PO, Bankole IA, Akpede GO. Central nervous manifestation of Lassa fever and the effect on mortality. J Neurol Sci. 2013;333(1):e604.

5 Solbrig MV, McCormick JB. Lassa fever: central nervous system manifestations. J Trop Goergr Neurol. 1991;1:23-30.

6 Madi D, Achappa B, Ramapuram JT, Chowta N, Laxman M, Mahalingam S. Dengue encephalitis-A rare manifestation of dengue fever. Asian Pac J Trop Biomed. 2014 May; 4 Suppl 1:S70-2.

7 Günther S, Weisner B, Roth A, Grewing T, Asper M, Drosten C, et al. Lassa fever encephalopathy: lassa virus in cerebrospinal fluid but not in serum. J Infect Dis. 2001 Aug;184(3):345-9.

8 Okoror LE, Esumeh FI, Agbonlahor DE, Umolu PI. Lassa virus: seroepidemiological survey of rodents caught in Ekpoma and environs. Trop Doct. 2005 Jan;35(1):16-7.

9 Dzotsi EK, Ohene SA, Asiedu-Bekoe F, Amankwa J, Sarkodie B, Adjabeng M, et al. The first cases of Lassa fever in Ghana. Ghana Med J. 2012 Sep;46(3):166-70.

10 Okokhere P, Asogun D, Okogbenin SA. The effect of malaria on the outcome of Lassa fever (Abstract). Int ] Infect Dis. 2010;14:e333.

11 Dongo AE, Kesieme EB, Iyamu CE, Okokhere PO, Akhuemokhan OC, Akpede GO. Lassa fever presenting as acute abdomen: a case series. Virol J. 2013 Apr;10(1):123.

12 Demby AH, Chamberlain J, Brown DW, Clegg CS. Early diagnosis of Lassa fever by reverse transcription-PCR. J Clin Microbiol. 1994 Dec;32(12):2898-903.

13 Ibekwe TS, Okokhere PO, Asogun D, Blackie FF, Nwegbu MM, Wahab KW, et al. Early-onset sensorineural hearing loss in Lassa fever. Eur Arch Otorhinolaryngol. 2011 Feb;268(2):197-201.

14 Cummins D, Bennett D, Fisher-Hoch SP, Farrar B, Machin SJ, McCormick JB. Lassa fever encephalopathy: clinical and laboratory findings. J Trop Med Hyg. 1992 Jun;95(3):197-201.

15 Jacobs M, Rodger A, Bell DJ, Bhagani S, Cropley I, Filipe A, et al. Late Ebola virus relapse causing meningoencephalitis: a case report. Lancet. 2016 Jul;388(10043):498-503. 
Okokhere et al.: Acute Lassa Virus Encephalitis with Lassa Virus in the CSF but Absent in the Blood

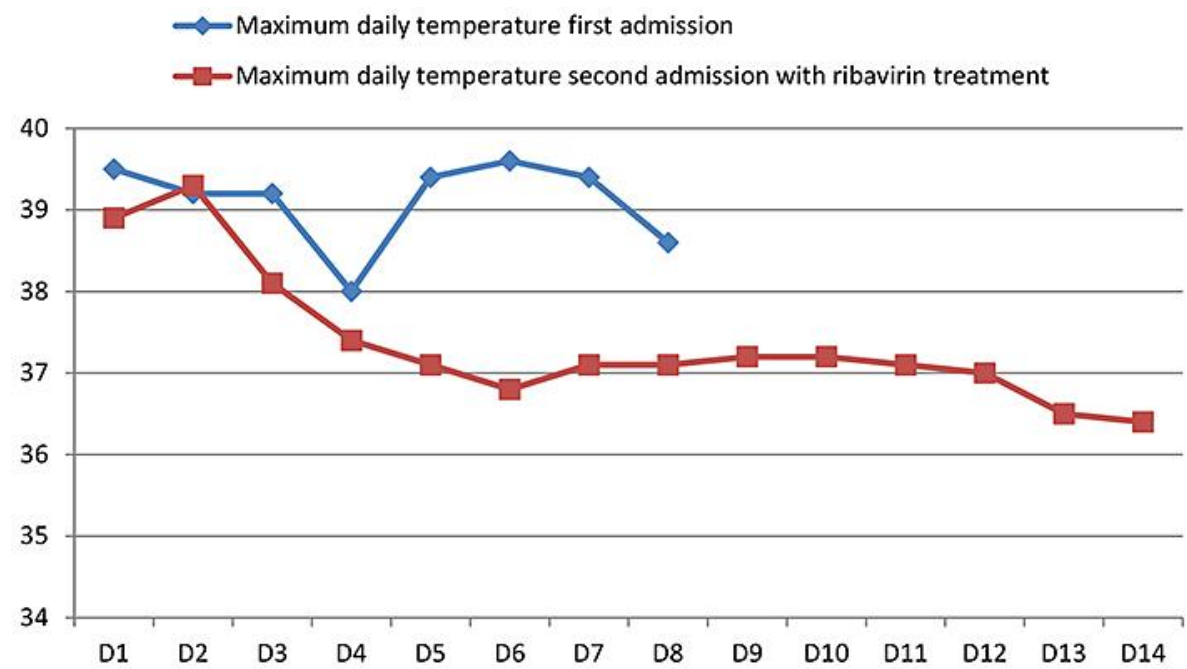

Fig. 1. Maximum daily temperature $\left({ }^{\circ} \mathrm{C}\right)$ during the first and second admissions.

Table 1. CSF and serum tests for evidence of Lassa virus (LAV) and viral antibodies

\begin{tabular}{lll}
\hline Parameter & Date & Result \\
\hline Blood RT-PCR for LAV & $27 / 12 / 2013$ & Negative \\
Blood RT-PCR for LAV & $05 / 01 / 2014$ & Negative \\
Serum IgM (ELISA) & $06 / 01 / 2014$ & Positive \\
Serum IgG (ELISA) & $06 / 01 / 2014$ & Positive \\
CSF RT-PCR for LAV & $07 / 01 / 2014$ & Positive \\
Post-treatment CSF RT-PCR for LAV & $28 / 01 / 2014$ & Negative \\
\hline
\end{tabular}


Table 2. Results of other tests conducted

\begin{tabular}{|c|c|c|}
\hline Parameter & $\begin{array}{l}\text { Result } \\
27 / 12 / 2013\end{array}$ & $\begin{array}{l}\text { Result } \\
05 / 01 / 2014\end{array}$ \\
\hline \multicolumn{3}{|l|}{ Complete blood count plus ESR } \\
\hline Pack cell volume (PCV), \% & 45.6 & 45.4 \\
\hline Haemoglobin, g/dL & 15.7 & 15.7 \\
\hline Total white blood cell count (TWBC), $\times 10^{9 / L}$ & 4.3 & 16.8 \\
\hline Neutrophil count, \% of TWBC & 81.5 & 43.4 \\
\hline Lymphocyte count, \% of TWBC & 9.8 & 46.3 \\
\hline Platelet count, $\times 10^{9} / \mathrm{L}$ & 122 & 210 \\
\hline Erythrocyte sedimentation rate (ESR), $\mathrm{mm} / \mathrm{h}$ & 12 & 67 \\
\hline \multicolumn{3}{|l|}{ Liver function test } \\
\hline Alkaline phosphatase, IU/L & ND & $132(9-35)$ \\
\hline Alanine aminotransferase, IU/L & ND & $69(\leq 12)$ \\
\hline Aspartate aminotransferase, IU/L & ND & $154(\leq 12)$ \\
\hline Total bilirubin, mg/dL & ND & $1.0(\leq 0.5)$ \\
\hline Conj. bilirubin, mg/dL & ND & $0.6(\leq 0.5)$ \\
\hline \multicolumn{3}{|l|}{ Serum proteins } \\
\hline Total protein, mg/dL & ND & 6.7 \\
\hline Albumin, mg/dL & ND & 3.0 \\
\hline \multicolumn{3}{|l|}{ Serum electrolytes, urea, and creatinine } \\
\hline Sodium, mmol/L & 136 & 141 \\
\hline Potassium, mmol/L & 4.0 & 4.0 \\
\hline Urea, mg/dL & 23 & 20 \\
\hline Creatinine, $\mathrm{mg} / \mathrm{dL}$ & $\mathrm{N} / \mathrm{A}$ & 0.7 \\
\hline \multicolumn{3}{|l|}{ Urinalysis } \\
\hline $\mathrm{pH}$ & 5.0 & 6.0 \\
\hline Protein & ++ & +++ \\
\hline Blood & Nil & ++ \\
\hline Glucose & Nil & Nil \\
\hline Microscopy & ND & $\begin{array}{l}\text { Pus cell: } 0-1 \text {; RBC: } 2-4 \text {; } \\
\text { casts: nil }\end{array}$ \\
\hline Urine culture & ND & No bacterial growth \\
\hline
\end{tabular}

